\title{
Blind Image Restoration Based on Semi-Causal Image Models
}

\author{
Naoyuki Fujiyama, Naoki Watanabe and Sueo Sugimoto \\ Department of Electrical and Electronic Engineering, Ritsumeikan University \\ Noji-Higashi, Kusatsu City, Shiga 525-8577 Japan \\ Tel: +81-(0)77-561-2673, FAX: +81-(0)77-561-2663 \\ e-mail: sugimoto@se.ritsumei.ac.jp
}

\begin{abstract}
In this paper, the image identification and restoration algorithm using only observable noisy blurred image based on the so-called semi-causal models is discussed. Our image identification is performed by applying the EM algorithm which is carried out by using the Kalman smoother estimates effectively. Also, we show compensation algorithms due to the approximation of Toeplitz matrices into circulant matrices in our image models.
\end{abstract}

\section{Introduction}

Blind image restoration is the process of estimating both the original image and the blur from the degraded image characteristics, using partial information about the imaging system [1]. In classical linear image restoration, the blurring function is given, and the degradation process is inverted using one of the many known restoration algorithms $[2,3]$. Unfortunately, in many practical situations, the blur is often unknown, and little information is available about the true image. Therefore, the original image must be identified directly from the observed image by using partial or. no information about the blurring process.

In this paper, the recursive Kalman filtering and smoothing algorithms are derived to enhance noisy blurred image data with the parameter identification scheme applying the EM algorithm [4], which can be effectively carried out by using Kalman smoothed estimators [5], for the so-called semi-causal random image models [6-9]. The advantage of the proposed method is that the restored image can be directly obtained from the noisy corrupted blur image without a priori statistical information on original image fields as well as knowledge of blurring functions (or point spread functions). Also, the blurring function, addtive noise in observed blur images and unknown parameters in the stochastic semi-causal model can be identified.

Define the index sets of $I_{x}$ and $J_{y}$ respectively as $I_{x} \equiv\{1, \cdots, M\}, J_{y} \equiv\{1, \cdots, N\}$. We assume the following statistical one-side-neighbor semi-causal model [6-9] for the image gray level $u(i, j)$ at site $(i, j)$ for $i \in I_{x}$ and $j \in J_{y}$.

$$
\begin{aligned}
u(i, j)= & \alpha_{0}\{u(i+1, j)+u(i-1, j)\} \\
& +\alpha_{1}\{u(i+1, j-1)+u(i-1, j-1)\} \\
& +\gamma u(i, j-1)+\xi(i, j),
\end{aligned}
$$

where $\alpha_{0}, \alpha_{1}$ and $\gamma$ are unknown constants and $\xi(i, j)$ is assumed as a Gaussian white noise sequence with $E[\xi(i, j)]=0$ and $E\left[\xi(i, j) \xi\left(i^{\prime}, j^{\prime}\right)\right]=\sigma_{\xi}^{2} \delta_{i-i^{\prime}} \delta_{j-j^{\prime}}$, where $\sigma_{\xi}^{2}$ is an unknown constant. $\delta$ is the Kronecker's delta function and $E[\cdot]$ denotes the expectation of ".".

Also noisy blurred observed image $z(i, j)$ is given by

$$
z(i, j)=\sum_{k=-K}^{K} \sum_{l=-L}^{L} h_{k, l} u(i-k, j-l)+\eta(i, j),
$$

where $\eta(i, j)$ is the Gaussian white noise disturbance with $E[\eta(i, j)]=0$ and $E\left[\eta(i, j) \eta\left(i^{\prime}, j^{\prime}\right)\right]=$ $\sigma_{\eta}^{2} \delta_{i-i^{\prime}} \delta_{j-j^{\prime}}$. Also $h_{k, l}$ is a blurring function. The degraded image is blurred in $(2 K+1) \times(2 L+1)$ sites by assuming $h_{k, l}<\varepsilon$ (: sufficiently small values) as $h_{k, l}=0$.

We now refine our image restoration problem in this paper as follows:

Estimate the original image $\{u(i, j), i \in$ $\left.I_{x}, j \in I_{x}\right\}$ and the unknown parameter set $\left\{\alpha_{0}, \alpha_{1}, \gamma, \sigma_{\xi}^{2}, \sigma_{\eta}^{2},\left\{h_{k, l}\right\}\right\}$, by using only given noisy blurred image $\left\{z(i, j), i \in I_{x}, j \in J_{y}\right\}$.

Let us rewrite the image and observation equations (1) and (2) in the vector-matrix representation as follows,

$$
\begin{aligned}
\mathbf{u}(j)= & \alpha_{0} \tilde{A} \mathbf{u}(j)+\alpha_{1} \tilde{A} \mathbf{u}(j-1) \\
& +\gamma I \mathbf{u}(j-1)+\boldsymbol{\xi}(j), \\
\mathbf{z}(j)= & \sum_{l=-L}^{L} \tilde{H}_{l} \mathbf{u}(j-l)+\eta(j),
\end{aligned}
$$


for $j \in J_{y}$, where $I$ is a $M \times M$ unit matrix, and

$$
\begin{aligned}
& \tilde{A} \equiv\left[\begin{array}{cccc}
0 & 1 & & 0 \\
1 & \ddots & \ddots & \\
& \ddots & \ddots & 1 \\
0 & & 1 & 0
\end{array}\right] \\
& \tilde{H}_{l} \equiv\left[\begin{array}{cccccc}
h_{0, l} & h_{-1, l} & \ldots & h_{-K, l} & & 0 \\
h_{1, l} & \ddots & \ddots & & \ddots & \\
\vdots & \ddots & \ddots & \ddots & & h_{-K, l} \\
h_{K, l} & & \ddots & \ddots & \ddots & \vdots \\
& \ddots & & \ddots & \ddots & h_{-1, l} \\
0 & & h_{K, l} & \cdots & h_{1, l} & h_{0, l}
\end{array}\right], \\
& \mathbf{u}(j) \equiv[u(1, j), \cdots, u(M, j)]^{T}, \\
& \mathbf{z}(j) \equiv[z(1, j), \cdots, z(M, j)]^{T}, \\
& \boldsymbol{\xi}(j) \equiv[\xi(1, j), \cdots, \xi(M, j)]^{T}, \\
& \eta(j) \equiv[\eta(1, j), \cdots, \eta(M, j)]^{T},
\end{aligned}
$$

where $T$ denotes the transpose of a vector or a matrix. Also $E[\xi(j)]=0, E[\eta(j)]=0$ and $\operatorname{Cov}[\xi(j)]=\sigma_{\xi}^{2} I$, $\operatorname{Cov}[\eta(j)]=\sigma_{\eta}^{2} I$, where $\operatorname{Cov}[\cdot]$ denotes the covariance of "."

\section{Identification and Restoration Algorithm}

\subsection{Circulant Matrix and FFT}

First, we approximate the Toepliz matrices $\tilde{A}$ and $\left\{\tilde{H}_{l}\right\}$ to the circulant matrices $A$ and $\left\{H_{l}\right\}$ as follows,

$$
\tilde{A} \simeq A \equiv\left[\begin{array}{ccccc}
0 & 1 & 0 & \cdots & 1 \\
1 & 0 & \ddots & \ddots & \vdots \\
0 & \ddots & \ddots & \ddots & 0 \\
\vdots & \ddots & \ddots & \ddots & 1 \\
1 & \cdots & 0 & 1 & 0
\end{array}\right]
$$

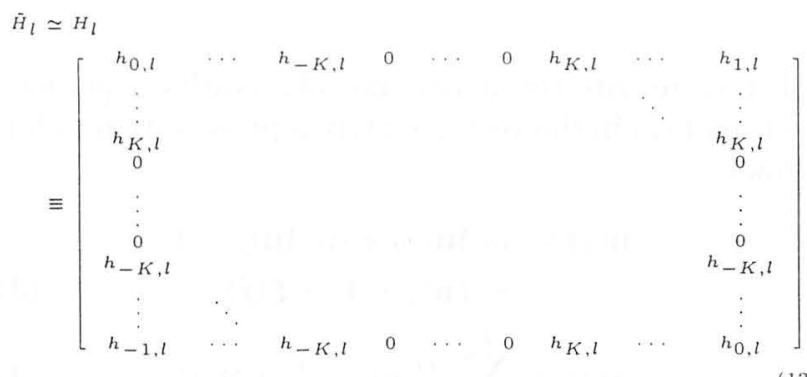

Then (3) and (4) are approximated by

$$
\begin{aligned}
& \mathbf{u}(j)=\alpha_{0} A \mathbf{u}(j)+\alpha_{1} A \mathbf{u}(j-1)+\gamma I \mathbf{u}(j-1)+\xi(j), \\
& \mathbf{z}(j)=\sum_{l=-L}^{L} H_{l} \mathbf{u}(j-l)+\eta(j) .
\end{aligned}
$$

Let us apply discrete Fourie transformation matrix $\mathcal{F}$ such that all circulant matrices $A,\left\{H_{l}\right\}$ are diagonalized as follows,

$$
\begin{aligned}
& \mathcal{F} A \mathcal{F}^{*}= \operatorname{diag}\left[2,2 \cos \frac{2 \pi}{M}, \cdots,\right. \\
&\left.2 \cos \frac{2 \pi(r-1)}{M}, \cdots, 2 \cos \frac{2 \pi(M-1)}{M}\right], \\
& \mathcal{F} H_{l} \mathcal{F}^{*}=\operatorname{diag}\left[\sum_{k=0}^{K} h_{k, l} \nu_{1}^{k}+\sum_{k=1}^{K} h_{k, l} \nu_{1}^{M-k},\right. \\
&\left.\cdots, \sum_{k=0}^{K} h_{k, l} \nu_{M}^{k}+\sum_{k=1}^{K} h_{k, l} \nu_{M}^{M-k}\right] \\
& \equiv \operatorname{diag}\left[\lambda_{1}^{\left(H_{l}\right)}, \lambda_{2}^{\left(H_{l}\right)}, \cdots, \lambda_{M}^{\left(H_{l}\right)}\right],
\end{aligned}
$$

where the superscript " $*$ " denotes the complex conjugate of a number, or the conjugate transpose of a vector or a matrix,

$$
\begin{gathered}
\mathcal{F}=\left[\mathcal{F}_{s}^{(r)}\right]=\left[\frac{1}{\sqrt{M}} \exp \left\{-j \frac{2 \pi(r-1)(s-1)}{M}\right\}\right] ; \\
s, r=1,2, \cdots, M, \\
\nu_{r}=\exp \left\{j \frac{2 \pi(r-1)}{M}\right\} ; r=1,2, \cdots, M
\end{gathered}
$$

and

$$
\lambda_{r}^{\left(H_{l}\right)}=\sum_{k=0}^{K} h_{k, r} \nu_{r}^{k}+\sum_{k=1}^{K} h_{k, r} \nu_{r}^{M-k}
$$

Remark Using $A$ and $\left\{H_{l}\right\},(1)$ and (2) are exactly expressed by

$$
\begin{aligned}
\mathbf{u}(j)= & \alpha_{0} A \mathbf{u}(j)+\alpha_{1} A \mathbf{u}(j-1)+\gamma I \mathbf{u}(j-1)+\xi(j) \\
& -\left\{\alpha_{0} \check{A} \mathbf{u}(j)+\alpha_{1} \check{A} \mathbf{u}(j-1)\right\}, \\
\mathbf{z}(j)= & \sum_{l=-L}^{L} H_{l} \mathbf{u}(j-l)+\eta(j)-\sum_{l=-L}^{L} \check{H}_{l} \mathbf{u}(j-l),
\end{aligned}
$$

where

$$
\begin{aligned}
\check{A} & \equiv A-\tilde{A} \\
& =\left[\begin{array}{cccc}
0 & \cdots & 0 & 1 \\
\vdots & \ddots & & 0 \\
0 & & & \vdots \\
1 & 0 & \cdots & 0
\end{array}\right],
\end{aligned}
$$




$$
\begin{aligned}
\check{H}_{l} & \equiv H_{l}-\tilde{H}_{l} \\
& =\left[\begin{array}{cccccc}
0 & \cdots & 0 & h_{K, l} & \cdots & h_{1, l} \\
\vdots & \ddots & & & \ddots & \vdots \\
0 & & & & & h_{K, l} \\
h_{-K, l} & & & & & 0 \\
\vdots & \ddots & & & & \vdots \\
h_{-1, l} & \cdots & h_{-K, l} & 0 & \cdots & 0
\end{array}\right] .
\end{aligned}
$$

First we derive the identification and estimation algorithm for the approximated noisy blurred model of (13) and (14).

Multiply $\mathcal{F}$ to (13) and (14), we have

$$
\begin{aligned}
\mathcal{F} \mathbf{u}(j)= & \alpha_{0} \mathcal{F} A \mathcal{F}^{*} \mathcal{F} \mathbf{u}(j)+\alpha_{1} \mathcal{F} A \mathcal{F}^{*} \mathcal{F} \mathbf{u}(j-1) \\
& +\gamma \mathcal{F} \mathbf{u}(j-1)+\mathcal{F} \xi(j), \\
\mathcal{F} \mathbf{y}(j)= & \sum_{l=-L}^{L} \mathcal{F} H_{l} \mathcal{F}^{*} \mathcal{F} \mathbf{x}(j-l)+\mathcal{F} \eta(j) ; j \in J_{y} .
\end{aligned}
$$

Then, we have

$$
\begin{aligned}
\mathbf{x}(j)= & {\left[I-\alpha_{0} \Lambda^{(A)}\right]^{-1}\left[\alpha_{1} \Lambda^{(A)}+\gamma I\right] \mathbf{x}(j-1) } \\
& +\left[I-\alpha_{0} \Lambda^{(A)}\right]^{-1} \mathbf{w}(j), \\
\mathrm{y}(j)= & \sum_{l=-L}^{L} \Lambda^{\left(H_{l}\right)} \mathrm{x}(j-l)+\mathrm{v}(j),
\end{aligned}
$$

where $\mathrm{x}(j) \equiv \mathcal{F} \mathbf{u}(j), \mathbf{y}(j) \equiv \mathcal{F} \mathbf{z}(j), \mathbf{w}(j) \equiv \mathcal{F} \boldsymbol{\xi}(j)$, $\mathrm{v}(j) \equiv \mathcal{F} \eta(j), \Lambda^{(A)} \equiv \mathcal{F} A \mathcal{F}^{*}$ and $\Lambda^{\left(H_{l}\right)} \equiv \mathcal{F} H_{l} \mathcal{F}^{*}$.

Namely, the following set of $\mathrm{M}$ decoupled scalar equations can be obtained

$$
\begin{aligned}
& x_{i}(j)=f_{i} x_{i}(j-1)+g_{i} w_{i}(j), \\
& y_{i}(j)=\sum_{l=-L}^{L} \lambda_{i, l} x_{i}(j-l)+v_{i}(j),
\end{aligned}
$$

for $i \in I_{x}$ and $j \in J_{y}$, where

$$
\begin{aligned}
f_{i} & \equiv \frac{2 \alpha_{1} \cos \frac{2 \pi(i-1)}{M}+\gamma}{1-2 \alpha_{0} \cos \frac{2 \pi(i-1)}{M}}, \\
g_{i} & \equiv \frac{1}{1-2 \alpha_{0} \cos \frac{2 \pi(i-1)}{M}}, \\
\lambda_{i, l} & \equiv h_{0, l}+2 \sum_{k=1}^{K} h_{k, l} \cos \frac{2 \pi(i-1) k}{M} .
\end{aligned}
$$

We note that our observation equation (30) contains total $2 L+1$ time-delay terms, and thus, we define the following augmented state vector $\mathbf{X}_{i}(j)$,

$$
\mathbf{X}_{i}(j) \equiv\left[x_{i}(j), x_{i}(j-1), \cdots, x_{i}(j-2 L)\right]^{T}
$$

By using the augmented state vector $\mathbf{X}_{i}(j),(29)$ and (30) can be written by the following vector-matrix equations,

$$
\begin{aligned}
\mathbf{X}_{i}(j) & =F_{i} \mathbf{X}_{i}(j-1)+G_{i} w_{i}(j), \\
y_{i}^{(p)}(j) & =\mathbf{h}_{i}^{T} \mathbf{X}_{i}(j)+v_{i}^{(p)}(j),
\end{aligned}
$$

for $i \in I_{x}$ and $j \in J_{y}$, where the $(2 L+1) \times(2 L+1)$ matrix $F_{i}$ is defined by

$$
F_{i} \equiv\left[\begin{array}{ccccc}
f_{i} & & & & 0 \\
1 & 0 & & & \\
0 & \ddots & \ddots & & \\
& \ddots & \ddots & \ddots & \\
0 & & 0 & 1 & 0
\end{array}\right],
$$

the $(2 L+1) \times 1$ vectors $G_{i}$ is defined by

$$
G_{i} \equiv\left[g_{i}, 0, \cdots, 0\right]^{T},
$$

the $1 \times(2 L+1)$ vectors $\mathbf{h}_{i}^{T}$ is defined by

$$
\mathbf{h}_{i}^{T} \equiv\left[\lambda_{i,-L}, \lambda_{i,-L+1}, \cdots, \lambda_{i, 0}, \cdots, \lambda_{i, L-1}, \lambda_{i, L}\right],(39)
$$

and $y_{i}^{(p)}(j), v_{i}^{(p)}(j)$ are defined by

$$
\begin{aligned}
& y_{i}^{(p)}(j) \equiv y_{i}(j-L), \\
& v_{i}^{(p)}(j) \equiv v_{i}(j-L) .
\end{aligned}
$$

Then, in the next subsection we will derive the algorithm to estimate the unknown parameters $\theta \equiv$ $\left[\alpha_{0}, \alpha_{1}, \gamma, \sigma_{\xi}^{2}, \sigma_{\eta}^{2},\left\{h_{k, l}\right\}\right]^{T}$ in our image models from only noisy blurred image $\left\{z(i, j), i \in I_{x}, j \in J_{y}\right\}$ based on the EM algorithm $[4,5]$.

\subsection{EM Algorithm}

The EM algorithm consists of two major steps: an expectation step, followed by a maximization step. The expectation is with respect to the unknown underlying variables, using the current estimate of the parameters and conditioned upon the observations. The maximization step then provides a new estimate of the parameters. These two steps are iterated until convergence.

We define the following sets,

$$
\left\{\mathbf{X}_{i}(0), \cdots, \mathbf{X}_{i}(N), y_{i}^{(p)}(1), \cdots, y_{i}^{(p)}(N)\right\},
$$

called the "complete data" involving the unknown underlying variables and

$$
\left\{y_{i}^{(p)}(1), \cdots, y_{i}^{(p)}(N)\right\},
$$


called the "incomplete data" consists of observation data.

Then the $\log$ likelihood function $\ln L_{i}(\theta)$ for the complete data can be given by

$$
\begin{aligned}
\ln L_{i}(\boldsymbol{\theta}) & \\
\equiv- & \frac{1}{2} \log (2 \pi)^{2 L+1}\left|\Sigma_{i}\right|-\frac{1}{2}\left\{\mathbf{X}_{i}(0)-\hat{\mathbf{X}}_{i}(0)\right\}^{*} \\
& \times \Sigma_{i}^{-1}\left\{\mathbf{X}_{i}(0)-\hat{\mathbf{X}}_{i}(0)\right\} \\
- & \frac{N}{2} \log \left(2 \pi g_{i}^{2} \sigma_{\xi}^{2}\right)-\frac{1}{2} \sum_{j=1}^{N}\left\{x_{i}(j)-f_{i} x_{i}(j-1)\right\}^{*} \\
& \times\left(g_{i}^{2} \sigma_{\xi}^{2}\right)^{-1}\left\{x_{i}(j)-f_{i} x_{i}(j-1)\right\} \\
- & \frac{N}{2} \log \left(2 \pi \sigma_{\eta}^{2}\right)-\frac{1}{2} \sum_{j=1}^{N}\left\{y_{i}^{(p)}(j)-\mathbf{h}_{i}^{T} \mathbf{X}_{i}(j)\right\}^{*} \\
& \times\left(\sigma_{\eta}^{2}\right)^{-1}\left\{y_{i}^{(p)}(j)-\mathbf{h}_{i}^{T} \mathbf{X}_{i}(j)\right\} .
\end{aligned}
$$

\subsubsection{E-step}

The expectation $Q_{i}\left(\boldsymbol{\theta} \mid \boldsymbol{\theta}^{(\mu)}\right)$ of (42) for the incomplete data and the estimate $\theta^{(\mu)}$ at the $\mu$-th iteration step, can be written as follows.

$$
\begin{aligned}
& Q_{i}\left(\boldsymbol{\theta} \mid \theta^{(\mu)}\right) \\
& \equiv E\left[\ln L_{i}(\theta) \mid y_{i}^{(p)}(1), \cdots, y_{i}^{(p)}(N), \theta^{(\mu)}\right] \\
& =(\text { constant })-\frac{N}{2} \log g_{i}^{2} \sigma_{\xi}^{2} \\
& \quad-\frac{1}{2 g_{i}^{2} \sigma_{\xi}^{2}}\left(a_{i} f_{i}^{2}-b_{i} f_{i}-b_{i}^{*} f_{i}+c_{i}\right) \\
& \quad-\frac{N}{2} \log \sigma_{\eta}^{2} \\
& \quad-\frac{1}{2 \sigma_{\eta}^{2}}\left(\mathbf{h}_{i}^{T} C_{i} \mathbf{h}_{i}-\mathbf{d}_{i}^{*} \mathbf{h}_{i}-\mathbf{h}_{i}^{T} \mathbf{d}_{i}+t_{i}\right),
\end{aligned}
$$

for $i \in I_{x}$, where

$$
\begin{aligned}
a_{i} & \equiv \sum_{j=1}^{N}\left\{P_{i}^{(1,1)}(j-1 \mid N)+\hat{x}_{i}(j-1 \mid N) \hat{x}_{i}^{*}(j-1 \mid N)\right\} \\
b_{i} & \equiv \sum_{j=1}^{N}\left\{P_{i}^{(1,1)}(j, j-1 \mid N)+\hat{x}_{i}(j \mid N) \hat{x}_{i}^{*}(j-1 \mid N)\right\}
\end{aligned}
$$

$$
\begin{aligned}
c_{i} & \equiv \sum_{j=1}^{N}\left\{P_{i}^{(1,1)}(j \mid N)+\hat{x}_{i}(j \mid N) \hat{x}_{i}^{*}(j \mid N)\right\}, \\
C_{i} & \equiv \sum_{j=1}^{N}\left\{P_{i}(j \mid N)+\hat{\mathbf{X}}_{i}(j \mid N) \hat{\mathbf{X}}_{i}^{*}(j \mid N)\right\},
\end{aligned}
$$

$$
\begin{aligned}
\mathrm{d}_{i}^{*} & \equiv \sum_{j=1}^{N} y_{i}^{(p)}(j) \hat{\mathbf{X}}_{i}^{*}(j \mid N), \\
t_{i} & \equiv \sum_{j=1}^{N} y_{i}^{(p)}(j) y_{i}^{(p) *}(j) .
\end{aligned}
$$

The superscript $(1,1)$ represents the $(1,1)$ element of the matrix and $\hat{\mathbf{X}}_{i}(j \mid N)$ is given by

$$
\begin{aligned}
\hat{\mathbf{X}}_{i}(j \mid N)=\left[\hat{x}_{i}(j \mid N), \hat{x}_{i}(j-1 \mid N),\right. & \cdots, \\
& \left.\hat{x}_{i}(j-2 L \mid N)\right]^{T} .
\end{aligned}
$$

(51) can be computed by applying the Kalman filter and fixed-interval smoothing algorithms as follows.

$$
\begin{aligned}
\hat{\mathbf{X}}_{i}(j \mid j-1)= & F_{i} \hat{\mathbf{X}}_{i}(j-1 \mid j-1) \\
\hat{\mathbf{X}}_{i}(j \mid j)= & \hat{\mathbf{X}}_{i}(j \mid j-1) \\
& +K_{i}(j)\left[y_{i}^{(p)}(j)-\mathbf{h}_{i}^{T} \hat{\mathbf{X}}_{i}(j \mid j-1)\right] \\
K_{i}(j)= & P_{i}(j \mid j-1) \\
& \times \mathbf{h}_{i}\left[\mathbf{h}_{i}^{T} P_{i}(j \mid j-1) \mathbf{h}_{i}+\sigma_{\eta}^{2}\right]^{-1} \\
P_{i}(j \mid j-1)= & F_{i} P_{i}(j-1 \mid j-1) F_{i}^{T}+\sigma_{\xi}^{2} G_{i} G_{i}^{T}, \\
P_{i}(j \mid j)= & P_{i}(j \mid j-1)-K_{i}(j) \mathbf{h}_{i}^{T} P_{i}(j \mid j-1)
\end{aligned}
$$

where we set $\hat{\mathbf{X}}_{i}(0 \mid 0)=0$ and $P_{i}(0 \mid 0)=\frac{g_{i}^{2} \sigma_{\xi}^{2}}{1-f_{i}^{2}} I$. In order to calculate $\hat{\mathbf{X}}_{i}(j \mid N)$ and $P_{i}(j \mid N)$, we carry out the backward recursions

$$
\begin{aligned}
\hat{\mathbf{X}}_{i}(j-1 \mid N)= & \hat{\mathbf{X}}_{i}(j-1 \mid j-1), \\
& +J_{i}(j-1)\left[\hat{\mathbf{X}}_{i}(j \mid N)-\hat{\mathbf{X}}_{i}(j \mid j-1)\right],(57) \\
J_{i}(j-1)= & P_{i}(j-1 \mid j-1) F_{i}^{T}\left[P_{i}(j \mid j-1)\right]^{-1}, \quad(58) \\
P_{i}(j-1 \mid N)= & P_{i}(j-1 \mid j-1)+J_{i}(j-1), \\
& \times\left[P_{i}(j \mid N)-P_{i}(j \mid j-1)\right] J_{i}^{T}(j-1),(59)
\end{aligned}
$$

for $j=N, N-1, \cdots, 1$. The covariance $P_{i}(j, j-$ $1 \mid N) \equiv E\left[\left\{\mathbf{X}_{i}(j \mid N)-\hat{\mathbf{X}}_{i}(j \mid N)\right\}\left\{\mathbf{X}_{i}(j-1 \mid N)-\hat{\mathbf{X}}_{i}(j-\right.\right.$ $\left.1 \mid N)\}^{*}\right]$ can be calculated using the backward recursions

$$
\begin{aligned}
P_{i}(j-1, j-2 \mid N)= & P_{i}(j-1 \mid j-1) J_{i}^{T}(j-2) \\
& +J_{i}(j-1)\left[P_{i}(j, j-1 \mid N)\right. \\
& \left.-F_{i} P_{i}(j-1 \mid j-1)\right] J_{i}^{T}(j-2),
\end{aligned}
$$

for $j=N, \cdots, 2$, where

$$
P_{i}(N, N-1 \mid N)=\left[I-K_{i}(N) \mathbf{h}_{i}^{T}\right] F_{i} P_{i}(N-1 \mid N-1) .
$$

\subsubsection{M-step}

The maximization of (44) yields

$$
\hat{f}_{i}=\frac{\Re\left[b_{i}\right]}{a_{i}},
$$




$$
\begin{gathered}
\widehat{g_{i}^{2} \sigma_{\xi_{i}}^{2}}=\frac{1}{N}\left[a_{i} \hat{f}_{i}^{2}-2 \Re\left[b_{i}\right] \hat{f}_{i}+c_{i}\right], \\
\hat{\mathbf{h}}_{i}^{T}=\Re\left[\mathbf{d}_{i}^{T}\right] \Re\left[C_{i}^{T}\right]^{-1}, \\
\hat{\sigma}_{\eta_{i}}^{2}=\frac{1}{N}\left[\hat{\mathbf{h}}_{i}^{T} \Re\left[C_{i}\right] \hat{\mathbf{h}}_{i}-2 \Re\left[\mathbf{d}_{i}^{*}\right] \hat{\mathbf{h}}_{i}+t_{i}\right],
\end{gathered}
$$

where $\Re[\cdot]$ denotes the real part of a complex number ". ".

Then, by using (31), (32) and (33), the unknown parameters $\hat{\alpha}_{0}, \hat{\alpha}_{1}, \hat{\gamma},\left\{\hat{h}_{k, l}\right\}$ can be estimated. First, we show the method in order to estimate the unknown parameters $\beta \equiv\left[\alpha_{0}, \alpha_{1}, \gamma\right]^{T}$ involved in the semi-causal model from the estimates $\left\{\hat{f}_{i}, i \in I_{x}\right\}$. We use the following least square error criterion,

$$
\hat{\boldsymbol{\beta}}=\arg \min _{\boldsymbol{\beta}} \sum_{i \in I_{s}}\left(f_{i}(\boldsymbol{\beta})-\hat{f}_{i}\right)^{2},
$$

where $I_{s}$ is the set satisfied with the stable condition $\left|\hat{f}_{i}\right|<1$. For solving (66), we apply Newton-Raphson method such that the initial values $\beta_{0}$ are chosen by as follows. The (31) is rewritten as

$$
\begin{gathered}
\hat{f}_{i}-d_{i} \alpha_{0} \hat{f}_{i}-d_{i} \alpha_{1}-\gamma \cong 0, \\
d_{i} \equiv 2 \cos \left(\frac{2 \pi(i-1)}{M}\right),
\end{gathered}
$$

where $i \in I_{s} \subset I_{x}$ is a set of the elements. Moreover, (67) can be rewritten as vector-matrix representation,

$$
D \beta=\hat{\mathrm{f}},
$$

for $s \in I_{s}$, where

$$
D=\left[\begin{array}{ccc}
\vdots & \vdots & \vdots \\
d_{s} \hat{f}_{s} & d_{s} & 1 \\
\vdots & \vdots & \vdots
\end{array}\right]
$$

and

$$
\hat{\mathbf{f}}=\left[\cdots, \hat{f}_{s}, \cdots\right]^{T}
$$

The initial values $\boldsymbol{\beta}_{0}$ is determined as follows,

$$
\boldsymbol{\beta}_{0}=\left(D^{T} D\right)^{-1} D^{T} \hat{\mathbf{f}} .
$$

Next the estimation method of $\hat{\sigma}_{\xi_{i}}^{2}$ from the estimate $\widehat{g_{i}^{2} \sigma_{\xi}^{2}}$ is represented as follows. Since $g_{i}$ can be computed by the estimate $\hat{\alpha}_{0}$ as follows,

$$
\hat{g}_{i}=\frac{1}{1-2 \hat{\alpha}_{0} \cos \frac{2 \pi(i-1)}{M}},
$$

we can obtain the estimate

$$
\hat{\sigma}_{\xi_{i}}^{2}=\frac{1}{N} \frac{1}{\hat{g}_{i}^{2}}\left[a_{i} \hat{f}_{i}^{2}+2 \Re\left[b_{i}\right] \hat{f}_{i}+c_{i}\right] \text {, }
$$

for $i=1, \cdots, M$. The final estimation $\hat{\sigma}_{\xi}^{2}$ can be obtained by the arithmetic average.

Similarly, we can estimate the unknown parameters $\left\{\hat{h}_{k, l}\right\}$ and $\hat{\sigma}_{\eta_{i}}^{2}$ involved in the obsevation equaiton. The relations between $\left\{h_{k, l}\right\}$ and $\left\{\lambda_{i, l}\right\}$ for $k=$ $-K, \cdots, 0, \cdots, K, l=-L, \cdots, 0, \cdots, L$ are shown as

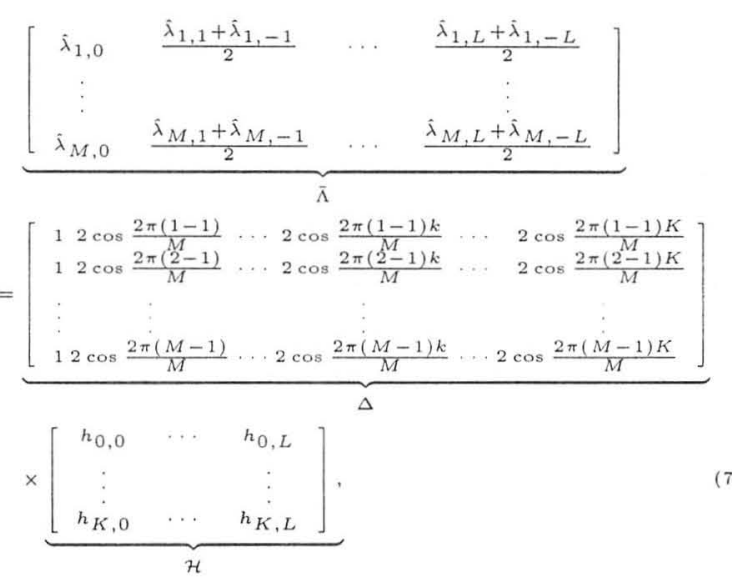

when we assume the symmetric property of $\left\{h_{k, l}\right\}$ as $h_{-k, l}=h_{k, l}=h_{k=-l}$ for $k=0, \cdots, K, l=0, \cdots, L$. Then the least square estimate $\hat{\mathcal{H}}$ is given as follows,

$$
\hat{\mathcal{H}}=\left(\Delta^{T} \Delta\right)^{-1} \Delta^{T} \bar{\Lambda}
$$

Also we can obtain the final estimate $\hat{\sigma}_{\eta}^{2}$ by the arithmetic average of $\left\{\hat{\sigma}_{\eta_{i}}^{2}\right\}, i=1, \cdots, M$, where (76) substitute into (65).

Compensation Algorithms Here, we discuss compensation algorithms due to the approximation of Toeplitz matrices $\tilde{A},\left\{\tilde{H}_{l}\right\}$ into circulant matricess $A$, $\left\{H_{l}\right\}$. Using (20) and (21), we can derive the equations corresponding to (29) and (30) as follows,

$$
\begin{aligned}
& x_{i}(j)=f_{i} x_{i}(j-1)-g_{i} \phi_{i}(j-1)+g_{i} w_{i}(j), \\
& y_{i}(j)=\sum_{l=-L}^{L} \lambda_{i, l} x_{i}(j-l)+v_{i}(j)-\psi_{i}(j),
\end{aligned}
$$


where

$$
\begin{aligned}
\phi_{i}(j-1) & \equiv \alpha_{0} \sum_{q=1}^{M}\left(\mathcal{F}_{M}^{(i)} \mathcal{F}_{1}^{(q) *}+\mathcal{F}_{M}^{(q)} \mathcal{F}_{1}^{(i) *}\right) x_{q}(j) \\
& +\alpha_{1} \sum_{q=1}^{M}\left(\mathcal{F}_{M}^{(i)} \mathcal{F}_{1}^{(q) *}+\mathcal{F}_{M}^{(q)} \mathcal{F}_{1}^{(i) *}\right) x_{q}(j-1), \\
\psi_{i}(j) & \equiv \sum_{l=-L}^{L}\left\{\sum _ { q = 1 } ^ { M } \left(\sum_{p=1}^{K} \sum_{k=p}^{K} h_{k, l} \mathcal{F}_{M-(k-p)}^{(i)} \mathcal{F}_{p}^{(q) *}\right.\right. \\
& \left.\left.+\sum_{p=1}^{K} \sum_{k=p}^{K} h_{k, l} \mathcal{F}_{M-(k-p)}^{(q)} \mathcal{F}_{p}^{(i) *}\right) x_{q}(j-l)\right\}
\end{aligned}
$$

Then, $\left\{\phi_{i}(j)\right\},\left\{\psi_{i}(j)\right\}$ are treated as known inputs $\left\{\hat{\phi}_{i}(j), \hat{\psi}_{i}(j)\right\}$ by using all estimates obtained previously under Toeplitz approximation of the circulant matrices so that we can apply again Kalman fixedinterval smoother algorithms to compensate the effect of the Toeplitz approximation.

Finally, we summarize our image processing algorithm as follows,

Step-1 Compute $\mathbf{y}(j)=\mathcal{F} \mathbf{z}(j), j \in J_{y}$.

Step-2 Give initial estimates $\theta^{(0)}$ in (1) and (2), and set $\mu=0$.

Step-3 Let $\mu=\mu+1$. Compute (62)-(65) using (45)(50), and Kalman filter and smoothing algorithms in (52)-(61).

Step-4 Estimate $\hat{\alpha}_{0}, \hat{\alpha}_{1}, \hat{\gamma}$ and $\hat{\sigma}_{\xi_{i}}^{2}$ from $\left\{\hat{f}_{i}\right\},\left\{\hat{g}_{i}\right\}$, using (66), (73) and (74).

Step-5 Estimate $\left\{\hat{h}_{k, l}\right\}$ from $\hat{\mathbf{h}}_{i}^{T}$, using (76).

Step-6 Estimate $\hat{\sigma}_{\eta_{i}}^{2}$ from $\left\{\hat{h}_{k, l}\right\}$, using (65), and improved estimates.

Step-7 Repeat to Step-6 from Step-3 until the estimated parameters are convergence.

Step-8 Using all estimates, obtain fixed-interval smoother $\hat{\mathbf{x}}_{i}$ for (77) and (78).

Step-9 Compute $\hat{\mathbf{u}}(j)=\mathcal{F}^{-1} \hat{\hat{\mathbf{x}}}(j \mid N), j \in J_{y}$.

\section{Conclusion}

In this paper, we have derived the iterative - recursive image identification and restoration algorithm, using only given noisy blurred image, based upon a semicausal random image models. In our algorithms, the
EM algorithm by using Kalman smoothed estimators is applied to estimating unknown parameters in our image models.

Furthermore compensation algorithms due to the approximation of Toepliz matrices into circulant matrices in our image models are discussed. We have shown that the compensated restoration images can be obtained by applying Kalman fixed-interval smoother algorithms with known inputs, which are all estimates obtained previously under Toeplitz approximation.

\section{References}

[1] D. Kundur and D. Hatzinakos, "Blind Image Deconvolution," IEEE Signal Processing Magazine, pp. 43-64, 1996.

[2] H. C. Andrews and B. R. Hunt, Digital Image Restoration, Prentice-Hall, New Jersey, 1977.

[3] A. K. Jain, Fundermentals of Digital Image Processing, Prentice Hall, New Jersey, 1989.

[4] A. P. Dempster N. M. Laird and D. B. Rubin, "Maximum Likelihood from Incomplete Data via the EM algorithm," J. Roy. Statis. Soc., vol. B39, pp.1-39, 1977.

[5] R. H. Shumway and D. S. Stoffer, "An Approach to Time Series Smoothing and Forecasting Using the EM Algorithm," J. Time Series Analysis, vol. 3, no. 4, pp. 253-264, 1982.

[6] A. K. Jain and J. R. Jain, "Partial Differential Equations and Finite Difference Methods in Image Processing Part II: Image Restoration," IEEE Trans. Autom. Control, vol. AC-23, no. 5, pp. 817-833, 1978.

[7] S. Sugimoto, H. Mizutani and T. Mizokawa, "Parameter Identification, Causality and Recursive Estimation for a Two-Dimensional Random Image Field," Proc. Int. Conf. on Cyber. and Soc., Denver, 1979.

[8] H. Mizutani and S. Sugimoto, "Algorithm of Two-Dimensional Image Identification and Restoration Based on an Extended Stochastic Semi-Causal Image Model," Trans. IECE Japan, Part A, vol. J64-A, no. 11, pp. 932-932, (in Japanese), 1981.

[9] S. Sugimoto, "Application to Image Processing," Chapter 2 in Stochastic Systems Theory III, ed. Y. Sunahara, Asakura Shoten, Tokyo, (in Japanese), 1982. 